\title{
PACIENTE CON PRIMER INGRESO EN LA UNIDAD DE TRASTORNOS DE LA CONDUCTA ALIMENTARIA. ANOREXIA NERVIOSA: ABORDAJE DE ENFERMERÍA
}

\section{NURIA SIMÓN ALONSO}

Enfermera residente en Salud Mental. Área de Salud Mental. Hospital de Salud Mental de Teruel.

\section{INTRODUCCIÓN}

Los problemas de la conducta alimentaria son toda entidad psicológica que se da en relación con la conducta de comer. Dentro de esta terminología, encontramos la anorexia y bulimia nerviosas, la vigorexia, la ingesta compulsiva y la ortorexia, entre otras ${ }^{1-6}$.

La anorexia nerviosa es una entidad clínica que se manifiesta por rechazo de la persona a mantener un peso corporal dentro de unos límites normales para su constitución y edad. Se acompaña de distorsión de la imagen corporal, fobia a la ganancia ponderal, alegría por perder peso y preocupación desmesurada por su físico ${ }^{1-6}$.

Actualmente, la quinta edición del Manual diagnóstico y estadístico de los trastornos mentales (DSM5) diferencia entre dos tipos de anorexia, de tipo restrictivo y de tipo compulsivo/purgativo ${ }^{4}$ :

- De tipo restrictivo: el paciente no recurre a atracones ni técnicas compensatorias (vómitos, laxantes, diuréticos, etc.).

Correspondencia: N. Simón Alonso

Correo electrónico: n.simonalonso@gmail.com
- De tipo compulsivo/purgativo: el paciente regularmente se provoca el vómito o tiene otras conductas similares tras la ingesta de alimentos, aun siendo en pequeñas cantidades ${ }^{4,6}$.

\section{PRESENTACIÓN DEL CASO}

Paciente con diagnóstico de anorexia nerviosa que ingresa en la unidad de trastornos de la conducta alimentaria derivado del hospital de día, debido a la imposibilidad de manejo en dicho dispositivo.

\section{Historia de la enfermedad}

El paciente comenta que en el colegio cogió un poco de peso a los 10 años, y que algún compañero le llamaba «tetitas», por lo que se apuntó a fútbol y en casa hacía abdominales. A los 12 años, empezó a hacer más ejercicio, comía menos «chuches», helados y bollería, tras lo cual, perdió 3-4 kg. Dado que la gente decía que estaba delgado, dejó de hacer tantos abdominales, pero restringió completamente los helados, las pizzas y lo que consideraba que no era «sano». Siguió perdiendo peso, y dejó el fútbol. Con 13 años, ya no comía bocadillos; a veces, se 
veía demasiado delgado, pero prefería las bromas con respecto a la delgadez que las bromas relacionadas con el sobrepeso. Sus padres empezaron a preocuparse, acudieron a la pediatra y recibieron pautas de actuación. Los primeros días comía un poco más, pero después siguió comiendo igual, sin helados ni bollería. Dejó de merendar o merendaba fruta.

\section{Exploración física al ingreso en la unidad}

Peso: 44,8 kg. Talla: 1,70 m. Índice de masa corporal: $15,5 \mathrm{~kg} / \mathrm{m}^{2}$.

\section{VALORACIÓN DE ENFERMERÍA MEDIANTE LOS PATRONES DE MARJORY GORDON}

Se realiza una rigurosa valoración enfermera de salud mental basándonos en los patrones funcionales de Marjory Gordon (tabla 1) 7 .

\section{DESCRIPCIÓN DE DIAGNÓSTICOS Y PLANIFICACIÓN DE CUIDADOS}

Se utiliza la taxonomía NANDA (North American Nursing Diagnosis Association) 2015-20178 para

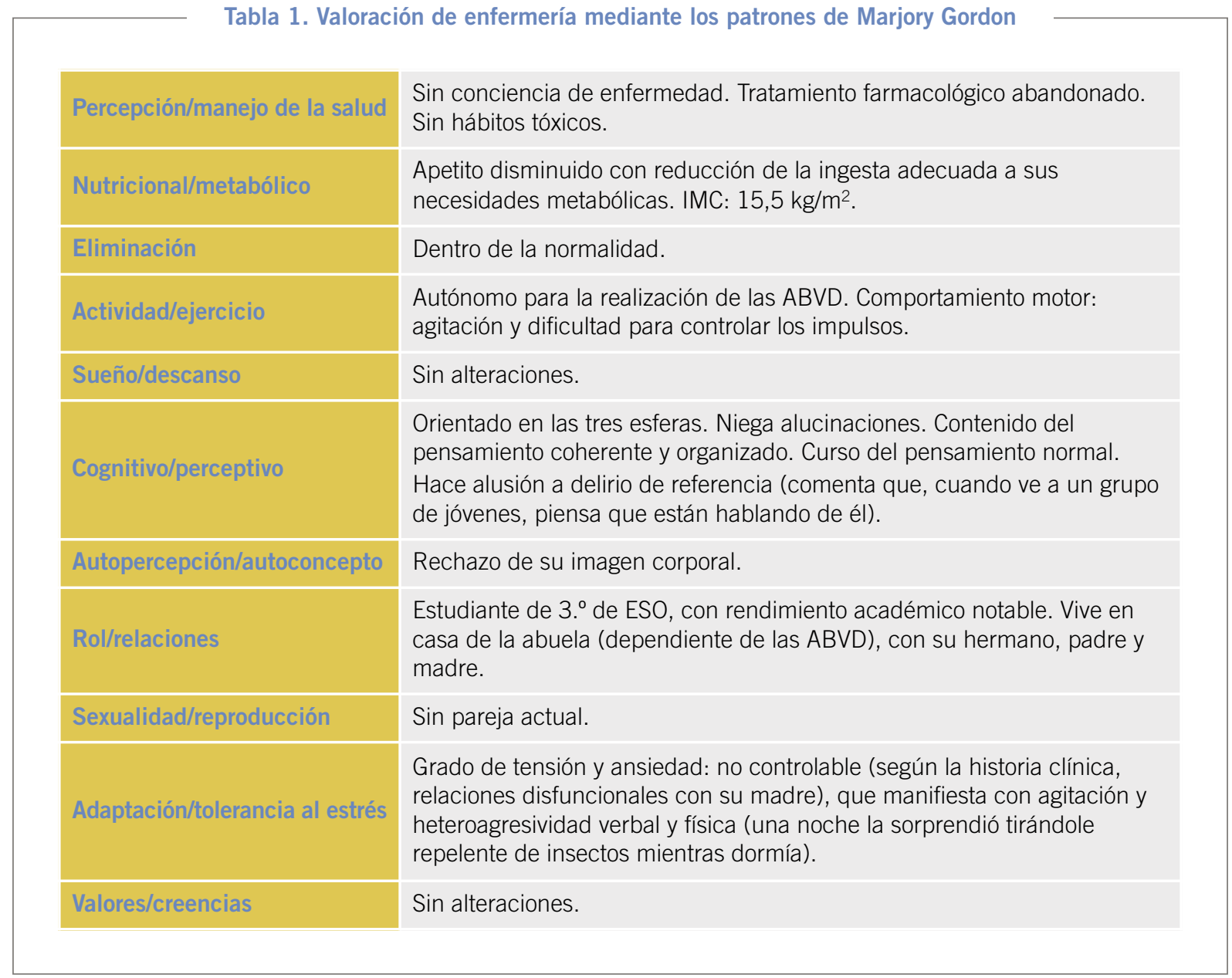

ABVD: actividades básicas de la vida diaria; ESO: Educación Secundaria Obligatoria; IMC: índice de masa corporal. 
formular los diagnósticos de enfermería, y la clasificación de resultados NOC (5. ${ }^{a}$ edición $)^{9}$ y la clasificación de intervenciones NIC (6. ${ }^{a}$ edición) ${ }^{10}$ para la elaboración del plan de cuidados. Los indicadores de los resultados de cada diagnóstico se acompañan de tres mediciones (PV: puntuación en la valo- ración; PI: puntuación ideal; y PA: puntuación al alta). Para evaluar los criterios de resultado, se ha utilizado una escala de tipo Likert, que oscila de 1 (la peor puntuación posible) a 5 (la máxima puntuación alcanzable) (tabla 2).

Tabla 2. Diagnósticos (NANDA), objetivos (NOC) e intervenciones (NIC) de enfermería

NANDA (00099) Mantenimiento ineficaz de la salud $\mathrm{r} / \mathrm{c}$ alteración de la función cognitiva m/p incapacidad para asumir la responsabilidad de llevar a cabo prácticas básicas de salud.

(1603) Conducta de búsqueda de salud. Indicador:

NOC

- 16006 Describe estrategias para eliminar la conducta poco saludable.

PV: 1 ; PI: 5; PA: 5

- 160308 Realiza conducta sanitaria prescrita.

(6160) Intervención en casos de crisis. Actividades:

- Proporcionar un ambiente de apoyo.

- Instaurar las precauciones necesarias para salvaguardar al paciente u otras personas con riesgo de lesiones físicas.

(4360) Modificación de la conducta. Actividades:

- Determinar la motivación del paciente para un cambio de conducta.

- Fomentar la sustitución de hábitos indeseables por otros deseables.

NANDA (00002) Desequilibrio nutricional: inferior a las necesidades corporales $\mathrm{r} / \mathrm{c}$ ingesta diaria insuficiente $\mathrm{m} / \mathrm{p}$ peso corporal inferior en un $20 \%$ o más respecto al rango de peso ideal.

(1626) Conducta de aumento de peso. Indicador:

- 162604 Establece objetivos de peso alcanzables.

PV: $1 ; \mathbf{P I}: 5 ; \mathbf{P A :} 4$

NOC (1622) Conducta de cumplimiento: dieta prescrita. Indicador:

- 162201 Participa en el establecimiento de objetivos dietéticos alcanzables.

PV: 1; PI: 5; PA: 5.

(5246) Asesoramiento nutricional. Actividades:

- Determinar la ingesta y los hábitos alimentarios del paciente.

- Facilitar la identificación de las conductas alimentarias que desea cambiar.

NIC

(5614). Enseñanza: Dieta prescrita. Actividades:

- Evaluar el nivel de conocimientos del paciente acerca de la dieta prescrita.

- Explicar el propósito del seguimiento de la dieta para la salud general. 
Tabla 2. Diagnósticos (NANDA), objetivos (NOC) e intervenciones (NIC) de enfermería (cont.)

NANDA (00118) Trastorno de la imagen corporal r/c alteración de la autopercepción m/p alteración de la visión del propio cuerpo.

(1200) Imagen corporal. Indicador:

NOC - Congruencia entre realidad corporal, ideal corporal e imagen corporal.

- Satisfacción con el aspecto corporal.

PV: 1; PI: 5; PA: 4

PV: 1 ; PI: 5; PA: 4

(5220) Mejora de la imagen corporal. Actividades:

NIC - Utilizar una orientación anticipatoria en la preparación del paciente para los cambios de imagen corporal que sean previsibles.

- Ayudar al paciente a desarrollar unas expectativas de imagen corporal más realistas.

NANDA (00146) Ansiedad r/c crisis situacional m/p inquietud y nerviosismo.

(1211) Nivel de ansiedad. Indicador:

\begin{tabular}{l|l|l} 
NOC & -121105 Inquietud. & PV: 1; PI: 5; PA: 4.
\end{tabular}

- 121106 Tensión muscular. $\quad$ PV: 1; PI: 5; PA: 3.

(5802) Disminución de la ansiedad. Actividades:

NIC - Permanecer con el paciente para promover la seguridad y reducir el miedo.

- Instruir en técnicas de relajación.

- Escuchar con atención.

NANDA (00069) Afrontamiento ineficaz $\mathrm{r} / \mathrm{c}$ sentido de control insuficiente $\mathrm{m} / \mathrm{p}$ estrategias de afrontamiento ineficaces.

(1302) Afrontamiento de problemas. Indicador:

NOC $\quad-130201$ Identifica patrones de superación eficaces. $\quad$ PV: 1; PI: 5; PA: 3.

- 130215 Busca ayuda profesional de forma adecuada. $\quad$ PV: 1; PI: 5; PA: 3.

(5340) Presencia. Actividades:

- Mostrar actitud de aceptación.

NIC

- Comunicar oralmente empatía o comprensión por la experiencia que está pasado el paciente.

(4360) Modificación de la conducta. Actividades:

- Determinar la motivación del paciente para un cambio de conducta.

- Fomentar la sustitución de hábitos indeseables por otros deseables.

m/p: manifestado por; PA: puntuación al alta; PI: puntuación ideal; PV: puntuación en la valoración; r/c: relacionado con.

\section{EVALUACIÓN DEL PLAN DE CUIDADOS Y SEGUIMIENTO}

Durante el ingreso hospitalario, se recabó información para la realización de un análisis del trastorno, determinando los factores desencadenantes, los mantenedores y sus consecuencias.

Se realizó un contrato conductual con el paciente, acordándose una serie de objetivos y metas con- 
ductuales respecto a la conducta alimentaria y la ganancia ponderal. Se le premió con privilegios terapéuticos conforme iba ganando el peso estipulado (paseos por la unidad, llamadas telefónicas, meriendas y comidas fuera de la unidad, etc.). En dicho contrato, también se incluyó la asistencia a los grupos psicoeducativos con la psicóloga dos veces por semana.

Respecto a los diagnósticos planteados, se ha alcanzado el grado deseado de conocimiento respecto a los objetivos «1603 Conducta de búsqueda de salud» y «1622 Conducta de cumplimiento: dieta prescrita», objetivándolos en la práctica clínica mediante la adaptación a las normas y dinámicas de la unidad, cumpliendo correctamente con las pautas alimentarias indicadas por el personal y participando activamente en las actividades de la unidad.

Es precisa la continuidad de cuidados de enfermería a través del centro de salud mental/hospital de día para conseguir la puntuación ideal en los objetivos «1626 Conducta de aumento de peso», «1200 Imagen corporal», «1211 Nivel de ansiedad» y «1302 Afrontamiento de problemas», ya que el paciente presenta dificultades para afrontar de forma efectiva los problemas, lo que lleva a momentos de malestar emocional que repercuten sobre sus pensamientos acerca de la alimentación. En el momento del alta, todavía persiste distorsión de la imagen corporal y cierto miedo a la recaída.

Para concluir, podemos afirmar, basándonos en nuestra práctica clínica, que la anorexia nerviosa es una entidad en la que aparecen frecuentemente cogniciones irracionales sobre la alimentación, el peso y la figura corporal y que, una vez instaurado el trastorno alimentario, se mantienen por sí mismas, unidas a una baja autocrítica. Entre las técnicas que han resultado ser más eficaces en el tratamiento de estas enfermedades, se encuentra la terapia cognitivo-conductual, donde se busca la reorientación en la realidad y el trabajo a través de conductas (ganancia ponderal) y consecuencias (privilegios).

\section{BIBLIOGRAFÍA}

1. Grupo de trabajo de la Guía de Práctica Clínica sobre Trastornos de la Conducta Alimentaria. Guía de Práctica Clínica sobre Trastornos de la Conducta Alimentaria. Madrid: Plan de Calidad para el Sistema Nacional de Salud del Ministerio de Sanidad y Consumo. Agència d'Avaluació de Tecnologia i Recerca Mèdiques de Cataluña; 2009. Guías de Práctica Clínica en el SNS: AATRM Núm. 2006/05-01.

2. Nieto E, Casado A, García C, Peláez L. Guía de Cuidados de Enfermería Familiar y Comunitaria en los Trastornos de la Conducta Alimentaria. Madrid: Federación de Asociaciones de Enfermería Comunitaria y Atención Primaria; 2008.

3. Unikel C, Caballero A. Guía clínica para la atención de trastornos mentales: Guía clínica para trastornos de la conducta alimentaria. México D.F.: Instituto Nacional de Psiquiatría Ramón de la Fuente Muñiz; 2010.

4. American Psychiatric Association. DSM-5. Guía de consulta de los criterios diagnósticos del DSM-5: DSM$5^{\mathrm{TM}}$. Spanish edition of the desk reference to the diagnostic criteria from DSM-5TM. Madrid: Editorial Médica Panamericana; 2013.

5. Organización Mundial de la Salud. Guia de bolsillo de la clasificacion CIE-10. Clasificacion de los trastornos mentales y del comportamiento. Con glosario y criterios diagnosticos de investigacion. CIE-10: CDI-10. Madrid: Editorial Medica Panamericana; 2000.

6. Fornés J. Enfermería de salud mental y psiquiátrica. Valoración y cuidados. Madrid: Editorial Médica Panamericana; 2014.

7. Gordon M. Manual de diagnósticos enfermeros. 11. ${ }^{a}$ ed. Madrid: McGraw-Hill Interamericana de España S.L.; 2007.

8. NANDA International, Herdman TH, Kamitsuru S (eds). Diagnósticos enfermeros. Definiciones y clasificación 2015-2017. 10. a ed. Barcelona: Elsevier España, S.L.; 2015.

9. Moorhead S, Johnson M, Maas ML, Swanson E (eds.). Clasificación de Resultados de Enfermería (NOC): Medición de Resultados en Salud. 5. ${ }^{a}$ ed. Barcelona: Elsevier España, S.L.; 2014.

10. Bulechek GM, Butcher HK, Dochterman JM, Wagner C (eds.). Clasificación de Intervenciones de Enfermería (NIC). 6. ${ }^{a}$ ed. Barcelona: Elsevier España, S.L.; 2014. 